\title{
Estimação de parâmetros genéticos para escores visuais e características de desenvolvimento ponderal na raça Nelore
}

\author{
[Genetic parameter estimation for visual score and ponderal \\ development traits in Nellore cattle] \\ L.O. Duitama ${ }^{1}$, R. da Fonseca ${ }^{2}$, T. Bertipaglia ${ }^{1}$, C.H. Machado ${ }^{3}$, C.V. Soares Filho ${ }^{4}$ \\ ${ }^{1}$ Universidade Estadual Paulista - Faculdade de Ciências Agrárias e Veterinárias - Jaboticabal, SP \\ ${ }^{2}$ Universidade Estadual Paulista - Campus experimental de Dracena - Dracena, SP \\ ${ }^{3}$ Associação Brasileira dos Criadores de Zebu - Uberaba, MG \\ ${ }^{4}$ Universidade Estadual Paulista - Faculdade de Medicina Veterinária - Araçatuba, SP
}

\begin{abstract}
RESUMO
O objetivo foi avaliar a associação genética entre os escores visuais de estrutura, precocidade e musculosidade, com as características peso aos 18 meses, ganho de peso diário e o perímetro escrotal em machos Nelore. Foram utilizados 7.256 registros de animais participantes de provas de ganho de peso. As estimativas dos componentes de (co)variâncias foram obtidas por meio de Máxima Verossimilhança Restrita, empregando-se um modelo animal. Os efeitos fixos de grupo contemporâneo, idade e peso como covariável linear foram considerados; o único efeito aleatório foi o genético aditivo direto. As estimativas de herdabilidade de estrutura, precocidade e musculosidade foram: 0,$30 ; 0,37 ; 0,32$, respectivamente. As estimativas das correlações genéticas entre os escores variaram de 0,76 a 0,95 , indicando que os escores são controlados, em grande parte, pelo mesmo grupo de genes. As estimativas das correlações genéticas dos escores visuais com as características de ganho de peso diário, peso aos 18 meses e o perímetro escrotal apresentaram valores semelhantes entre os escores 0,45 a 0,$50 ; 0,80$ a 0,83 e $-0,05$ a $-0,03$, respectivamente; indicando que a seleção para os escores trará mudanças genéticas no mesmo sentido para o peso aos 18 meses e em menor medida para o ganho de peso.
\end{abstract}

Palavras-chave: estrutura corporal, musculosidade, precocidade, prova ganho de peso

\begin{abstract}
The aim of this study was to evaluate the genetic association between visual scores of structure, finishing precocity and muscling with weight at 18 months, daily weight gain and scrotal circumference in male Nellore. A total of 7,256 records of animals participating in weight gain trials were used. The (co)variance components were estimated by restricted maximum likelihood, under an animal model. Contemporary group, age and weight as covariate linears were included as fixed effects. Additive genetic effects were the only random effect. The heritability estimates for structure, precocity and muscling were $0.30 ; 0.37$ and 0.32 respectively. The genetic correlations between visual score were 0.76 to 0.95 , indicating that the visual scores are controlled by the same set of genes, a fact making individual selection more complex. The visual scores showed similar genetic correlations with daily weight gain, weight at 18 months and scrotal circumference, ranging from 0.45 to 0.50 , from 0.80 to 0.83 and from 0.05 to -0.03 , respectively, indicating that individual selection for visual scores will result in genetic changes in the same direction in weight at 18 months and, to a lesser extent, in daily weight gain.
\end{abstract}

Keywords: body structure, finishing precocity, muscling, weight gain trial

Recebido em 20 de maio de 2013

Aceito em 12 de março de 2015

E-mail: lorlando82@hotmail.com 


\section{INTRODUÇÃO}

Muitos dos programas de melhoramento genético ao redor do mundo perceberam que a inclusão de medidas de peso a diferentes idades nas avaliações genéticas não são suficientes para identificar aqueles animais que finalizam de forma precoce com boas características de carcaça. Por essa razão, começaram a incluir características morfológicas medidas de forma objetiva, como, por exemplo, medidas das dimensões corporais (Maiwashe et al., 2002; Afoloyan et al., 2007) ou medidas subjetivas por meio da atribuição visual de escores a um conjunto de atributos morfológicos (Ex. Musculosidade, precocidade) (Herring et al., 1994; Phöcas e Laloe, 2004; Boligon et al., 2011).

O Brasil é um dos países que mais têm trabalhado na inclusão de características morfológicas, avaliadas por meio de escores visuais, nos programas de melhoramento genético, procurando melhores resultados na identificação de biótipos economicamente mais eficientes, de acordo com suas condições ambientais. Como resultado das pesquisas, várias metodologias têm sido desenvolvidas, entre elas as mais utilizadas são: CPM, que avalia conformação, precocidade e musculosidade, e a metodologia EPM, que avalia estrutura, precocidade e musculosidade (Yokoo et al., 2009; Faria et al., 2009a; Koury Filho et al., 2009, 2010).

Com a utilização dos escores visuais, objetiva-se estimar a proporção dos diferentes tecidos da carcaça (músculo, osso, gordura) dos animais no momento do abate, e identificar animais de acabamento precoce (quantidade de gordura mínima necessária para o abate), evitando o aumento no peso adulto dos animais (Koury Filho et al., 2009).

As pesquisas realizadas mostram que existe variabilidade genética nos escores visuais, o que permitira progressos genéticos quando feita a seleção para essas características (Jorge Júnior et al., 2001; Toral et al., 2011). As vantagens apresentadas na avaliação, como o baixo custo e a facilidade de implementação, permitem que um grande número de animais possa ser avaliado. Uma desvantagem é a subjetividade dos escores, pois estes são obtidos por meio de interpretação do olho humano, abrindo espaço para interpretações pessoais (Cardoso et al., 2004).

A metodologia de avaliação visual EPM (Estrutura, Precocidade e Musculosidade) foi desenvolvida recentemente, e são poucos os trabalhos que estimaram parâmetros genéticos e sua relação com outras características de importância econômica, sendo necessários estudos que permitam definir se esses escores podem ser usados como critérios de seleção dentro dos programas de melhoramento genético animal. Nesse contexto, o objetivo deste trabalho foi avaliar a associação genética entre os escores visuais da metodologia EPM mensurados ao sobreano com as características peso aos 18 meses, ganho de peso diário e o perímetro escrotal em machos da raça Nelore.

\section{MATERIAL E MÉTODOS}

Os dados utilizados são provenientes de provas de ganho de peso a pasto de 7.256 machos Nelore, realizadas pela Associação Brasileira dos Criadores de Zebu (ABCZ) nos Estados de São Paulo, Paraná, Mato Grosso e Mato Grosso do Sul, entre os anos de 2003 e 2009. O regulamento usado pela $\mathrm{ABCZ}$ para a execução das provas de ganho de peso a pasto pode ser consultado no site http://www.pmgz.com.br/ ?pgpManual.

Foram avaliados ao final da prova (ao redor dos 18 meses de idade) os escores visuais de estrutura $(\mathrm{E})$, precocidade $(\mathrm{P})$ e musculosidade (M); ganho de peso médio diário da prova (GPD); peso aos 18 meses (P18) e o perímetro escrotal (PE). Os animais entraram na prova de ganho de peso com idades entre sete e dez meses, levando em conta que a máxima diferença de idade entre animais de um mesmo grupo não fosse maior que 90 dias, as condições de manejo durante a prova foram as mesmas para cada grupo. Após a entrada, começou o período de adaptação de 70 dias, com o objetivo de diminuir a influência de fatores ambientais e efeito materno do período pré-desmama sobre o desempenho dos animais na prova; depois desse período, iniciou-se a prova de ganho de peso, com duração de 224 dias.

A alimentação dos animais foi baseada em forragem do gênero Brachiaria spp., podendo ocorrer suplementação no período da seca, 
mistura mineral e água foram fornecidos à vontade.

O GPD e o P18 foram definidos como:

$$
\begin{aligned}
\mathrm{GDP}= & \frac{\mathrm{P}_{\mathrm{f}}-\mathrm{P}_{\mathrm{i}}}{\mathrm{D}_{\mathrm{p}}} \\
& \mathrm{P} 18=\mathrm{P}_{\mathrm{f}}+\left(\left(\mathrm{I}_{\mathrm{f}}-540\right) * \mathrm{GDP}\right)
\end{aligned}
$$

em que:

GPD = Ganho de peso médio diário da prova;

P18 = Peso aos 18 meses;

$\mathrm{P}_{\mathrm{f}}=$ Peso final da prova;

$\mathrm{P}_{\mathrm{i}}=$ Peso início da prova;

$\mathrm{D}_{\mathrm{p}}=$ Duração da prova em dias (224 dias);

$\mathrm{I}_{\mathrm{f}}=$ Idade final da prova.

As avaliações visuais foram feitas de forma individual utilizando a metodologia EPM (pontuação de um até seis) por técnicos treinados, de acordo com o seguinte procedimento: primeiramente observou-se todo o lote de animais a ser avaliado, o qual deve estar conformado por animais do mesmo sexo, mesma época de nascimento e submetidos às mesmas condições de alimentação e manejo; visualizouse o perfil médio do lote (escore três) para cada característica avaliada, que serviu de base de comparação; e finalmente atribuíram-se os escores a cada animal com referência ao grupo avaliado (perfil médio do grupo), existindo em cada grupo avaliado animais com todos os escores, sendo seis a maior expressão e um a menor expressão da característica (Faria et al., 2007). A distribuição esperada dos escores dentro de cada grupo avaliado e na população toda se aproxima a uma binomial com parâmetros $n=5$ e $p=0,5$.

Koury Filho et al. (2009) descreveram essas características como:

- Estrutura Corporal (E): Prediz visualmente a área que o animal abrange visto de lado, avaliando-se basicamente o comprimento corporal e a profundidade das costelas. Maiores áreas correspondem a maiores escores.

- Precocidade (P): Nessa avaliação, as maiores notas recaem sobre animais de maior profundidade de costelas em relação à profundidade de seus membros.

- Musculosidade (M): A musculosidade é avaliada pela evidência de massas musculares. Indivíduos mais grossos, com musculaturas mais convexas, recebem as maiores notas; e os mais finos, com musculatura menos convexa ou retilínea, as menores.

Para a preparação dos arquivos e a determinação dos efeitos fixos, foi usado o software R (R Core Team, 2010). Os efeitos de ambiente que influenciam cada característica foram determinados a partir de análises de variância prévia, usando a metodologia de quadrados mínimos. A Tabela 1 apresenta os efeitos fixos testados para cada característica e a significância do teste f com um $\alpha$ do $5 \%$.

Tabela 1. Valores de probalidade de $\mathrm{f} \operatorname{pr}(f)$ da análise de variância para os efeitos fixos das características de estrutura (E), precocidade (P), musculocidade (M), peso aos 18 meses (P18), ganho de peso diário (GPD) e perímetro escrotal (PE)

\begin{tabular}{lcccc}
\hline \multicolumn{1}{c}{ Característica } & $\mathrm{GC}^{1}$ & $\mathrm{P}_{\mathrm{f}}{ }^{2}$ & $\mathrm{I}_{\mathrm{f}}{ }^{* *}$ & $\mathrm{P}_{\mathrm{i}}{ }^{2}$ \\
\hline $\mathrm{E}$ & $<0,001^{* *}$ & $<0,001^{* * *}$ & $0,063^{\mathrm{ns}}$ & - \\
$\mathrm{P}$ & $<0,001^{* *}$ & $<0,001^{* * *}$ & $0,024^{* *}$ & - \\
$\mathrm{M}$ & $<0,001^{* *}$ & $<0,001^{* *}$ & $<0,001^{* *}$ & - \\
$\mathrm{GDP}$ & $<0,001^{* *}$ & - & - & $<0,001^{* *}$ \\
$\mathrm{PE}$ & $<0,001^{* *}$ & $<0,001^{* *}$ & $<0,001^{* *}$ & - \\
\hline
\end{tabular}

${ }^{1}$ Grupo contemporâneo.

${ }^{2}$ Covariável linear Peso final, Idade final e Peso inicial, respectivamente.

* e ${ }^{* *}$ Significativo ao 5 e $1 \%$ de probabilidade.

${ }^{\text {ns }}$ Não significativo.

Os animais participantes das provas de ganho de peso provinham de diferentes fazendas das quais não se tem informação detalhada do manejo; por tal razão, os grupos contemporâneos não consideraram informação anterior à prova, e foram definidos como os animais pertencentes a uma mesma prova de ganho de peso (animais com diferença de idade máxima de 90 dias, submetidos às mesmas condições de manejo durante o período de adaptação e da prova). Um 
dos propósitos do período de adaptação antes da execução da prova é diminuir a influência do manejo diferencial entre as fazendas.

O grupo contemporâneo apresentou-se significativo para todas as características. Para o P18, não se considerou nenhuma covariável, porque o valor usado já estava ajustado para a idade aos 540 dias; para a característica GPD, foi usada a covariável linear de peso ao início da prova, para corrigir as diferenças de peso existentes ao início da prova; as covariáveis lineares de idade e peso ao final da prova foram usadas para a característica de PE; para os escores visuais de E, P e M, as covariáveis de peso e idade ao final da prova se apresentaram significativas em todos, com exceção da idade para o escore de estrutura, porém foi muito próximo do nível de significância (Tab. 1).

Os critérios usados para a exclusão de registros foram os seguintes: Para as características GPD, P18 e PE, foram eliminadas observações com três desvios padrão acima ou abaixo da média do grupo contemporâneo, evitando assim a inclusão de outliers; para os escores visuais, não foi usado esse critério porque os escores atribuídos se encontram dentro dos limites um até seis, conforme a metodologia. Para as seis características, foram excluídos os grupos contemporâneos que continham apenas animais filhos de um mesmo touro, e aqueles com menos de cinco registros, para estimar de forma mais precisa os efeitos fixos.

A estimação dos componentes de (co)variância foi realizada pelo método da Máxima Verossimilhança Restrita (REML) com modelo linear, visto que Faria et al. (2008) concluíram que o modelo de limiar e linear podem ser usados na avaliação genética de escores visuais, sem que exista diferença significativa nas estimações.

Para a estimação, foi usado o software WOMBAT (Meyer, 2007) com uma estratégia de iteração que usa o algoritmo PX-EM (modificação do algoritmo EM) nas primeiras rodadas e, posteriormente, o algoritmo AI, diminuindo assim a sensibilidade que tem o último algoritmo aos valores inicias; a convergência foi atingida quando a mudança entre uma iteração e outra para o $\log$ da função de verossimilhança " $\log (L(\theta ; \mathrm{y}))$ " foi menor do que $1 \times 10^{-9}$, sendo reanudada a análise a cada convergência, usando como valores iniciais os obtidos na análise anterior. As características foram analisadas de forma unicaracterística e multicaracterísticas sob modelo animal. Em todas as análises foi utilizado um arquivo de genealogia contendo 20.364 animais, 1.949 touros e 11.157 mães na matriz de parentesco.

O modelo utilizado, em forma matricial para as características foi definido como segue.

$\mathrm{Y}=\mathrm{X} \beta+\mathrm{Za}+\mathrm{e}$

em que:

$\mathrm{Y}=$ vetor das observações de cada característica (E, P, M, GPD, P18 e PE);

$\mathrm{X}=$ matriz de incidência associada aos efeitos

fixos;

$\beta=$ vetor de solução para os efeitos fixos;

$\mathrm{Z}=$ matriz de incidência associada ao efeito

genético aditivo direto de cada animal;

$\mathrm{a}=$ vetor de soluções para os efeitos genéticos

aditivos diretos aleatórios;

e $=$ vetor dos resíduos

As pressuposições em relação aos componentes do modelo são:

$\mathrm{E}(\mathrm{y})=\mathrm{X} \beta, \mathrm{E}(\mathrm{a})=0$ e $\mathrm{E}(\mathrm{e})=0$

$$
\operatorname{var}\left[\begin{array}{l}
\mathrm{a} \\
\mathrm{e}
\end{array}\right]=\left[\begin{array}{ll}
\mathrm{G} & 0 \\
0 & \mathrm{R}
\end{array}\right]=\left[\begin{array}{cc}
\mathrm{G}_{0} \otimes \mathrm{A} & 0 \\
0 & \mathrm{R}_{0} \otimes \mathrm{I}_{\mathrm{n}}
\end{array}\right]
$$

em que:

$\mathrm{G}_{0}=$ matriz de (co)variâncias genética aditiva.

$\mathrm{R}_{0}=$ matriz de (co)variâncias dos efeitos

residuais.

$\mathrm{A}=$ matriz de parentesco.

$\mathrm{I}=$ matriz identidade.

$\otimes=$ operador de produto direto.

\section{RESULTADOS E DISCUSSÃO}

Na Tabela 2, é apresentada a estrutura dos dados analisados junto com as estatísticas descritivas para as seis características estudadas.

Para os escores visuais de E, P e M, a média e a moda apresentaram-se superiores ao esperado, uma vez que, segundo a metodologia de atribuição dos escores, estes deverão se assemelhar a uma distribuição normal em cada grupo avaliado, em cujo caso o valor esperado para os parâmetros de tendência central seria três. As medidas de desvio padrão e coeficiente de variação para os escores apresentaram valores 
inferiores ao reportado por Koury Filho et al. (2009) e Yokoo et al. (2009), indicando que nesse caso os escores estão mais concentrados em torno da média, apresentando frequências baixas nos escores extremos (Tab. 3).

Tabela 2. Estrutura do arquivo de dados analisados para as características de estrutura (E), precocidade (P) musculosidade $(\mathrm{M})$, ganho de peso diário (GPD-Kg), peso aos 18 meses (P18-Kg) e o perímetro escrotal (PE-cm)

\begin{tabular}{lcccccc}
\hline & $\mathrm{E}$ & $\mathrm{P}$ & $\mathrm{M}$ & GPD & $\mathrm{P} 18$ & $\mathrm{PE}$ \\
\hline $\mathrm{N}^{\text {o }}$ Animais & 7256 & 7256 & 7256 & 7253 & 7251 & 2026 \\
$\mathrm{~N}^{\text {o }}$ Mães & 6164 & 6164 & 6164 & 6161 & 6163 & 1818 \\
$\mathrm{~N}^{\text {o }}$ Touros & 755 & 755 & 755 & 755 & 755 & 289 \\
$\mathrm{NGC}$ & 153 & 153 & 153 & 153 & 153 & 53 \\
Média & 4,19 & 3,86 & 3,78 & 0,55 & 357,5 & 27,5 \\
Moda & 4 & 4 & 4 & - & - & - \\
D.P & 0,99 & 1 & 1 & 0,14 & 50,2 & 3,2 \\
$\mathrm{C} . \mathrm{V}^{3}$ & 23,6 & 25,9 & 26,4 & 25,4 & 13,3 & 11,6 \\
\hline
\end{tabular}

${ }^{1}$ Número de grupos contemporâneos.

${ }^{2}$ Desvio padrão.

${ }^{3}$ Coeficiente de variação.

Tabela 3. Frequência dos escores visuais em porcentagem (\%) para as características de estrutura (E), precocidade $(\mathrm{P})$ e musculosidade $(\mathrm{M})$

\begin{tabular}{c|c|ccccccc}
\hline \multirow{2}{*}{ Característica } & \multirow{2}{*}{ Número } & \multicolumn{7}{c}{ Escore } \\
\cline { 3 - 8 } & & 1 & 2 & 3 & 4 & 5 & 6 \\
\hline E & 7256 & 0,4 & 3,8 & 18,9 & 37,6 & 31,0 & 8,3 \\
P & 7256 & 0,3 & 7,4 & 28,9 & 36,8 & 21,8 & 4,8 \\
M & 7256 & 0,7 & 8,5 & 30,8 & 35,9 & 19,9 & 4,2 \\
\hline
\end{tabular}

As estimativas de herdabilidade para as características em estudo, sob análise multicaracterística, estão apresentadas na Tabela 4. De forma geral, os valores de herdabilidade e as variâncias genéticas e fenotípicas apresentaram-se semelhantes entre as análises unicaracterística e multicaracterística.

Para os escores visuais, foram testados vários modelos que diferiam na inclusão das covariáveis de peso e idade ao final da prova, que se mostraram significativas na análise de variância. O modelo que incluiu a co-variável linear de idade apresentou os menores erros de predição, sendo definido como o modelo mais adequado e o utilizado na análise final. Os maiores erros e menores valores de herdabilidade apresentaram-se quando foi adicionada a co- variável de peso, sugerindo que a correção por peso elimina parte da variância genética aditiva para os escores visuais. Possivelmente, isso é explicado pelo fato de os escores apresentarem alta associação genética com o peso, como se pode observar na Tabela 4.

As herdabilidades para os escores visuais de $\mathrm{E}, \mathrm{P}$ e M, de forma geral, apresentam-se semelhantes aos valores encontrados por Boligon et al. (2011) e Faria et al. (2010), que avaliaram os escores ao sobreano, e foram menores que os encontrados por Yokoo et al. (2009); entretanto, Toral et al. (2011) e Queiroz et al. (2011) acharam herdabilidades inferiores às reportadas neste trabalho. Todos os trabalhos foram realizados com dados da raça Nelore. 
Tabela 4. Estimativas da análise multicaracterística de variâncias genéticas $\left(\sigma_{\mathrm{a}}^{2}\right)$ e fenotípicas $\left(\sigma_{\mathrm{f}}^{2}\right)$, herdabilidades (diagonal principal), correlações genéticas (acima da diagonal) e correlações fenotípicas (abaixo da diagonal), com seus respectivos erros padrão em parêntesis, para as características de estrutura (E), precocidade $(\mathrm{P})$, musculosidade $(\mathrm{M})$, ganho de peso diário (GPD $-\mathrm{Kg}$ ), peso aos 18 meses (P18 $\mathrm{Kg})$ e o perímetro escrotal $(\mathrm{PE}-\mathrm{cm})$

\begin{tabular}{|c|c|c|c|c|c|c|}
\hline Car. ${ }^{1}$ & $\mathrm{E}$ & $\mathrm{P}$ & $\mathrm{M}$ & GPD & P18 & $\mathrm{PE}$ \\
\hline $\mathrm{E}$ & $0,30(0,04)$ & $0,77(0,04)$ & $0,76(0,04)$ & $0,45(0,09)$ & $0,83(0,04)$ & $-0,04(0,14)$ \\
\hline $\mathrm{P}$ & $0,56(0,01)$ & $0,37(0,04)$ & $0,95(0,02)$ & $0,50(0,08)$ & $0,81(0,04)$ & $-0,05(0,13)$ \\
\hline M & $0,60(0,01)$ & $0,72(0,01)$ & $0,32(0,04)$ & $0,46(0,08)$ & $0,80(0,04)$ & $-0,03(0,14)$ \\
\hline GPD & $0,27(0,01)$ & $0,33(0,01)$ & $0,34(0,01)$ & $0,26(0,04)$ & $0,78(0,04)$ & $-0,21(0,14)$ \\
\hline P18 & $0,60(0,01)$ & $0,56(0,01)$ & $0,59(0,01)$ & $0,83(0,01)$ & $0,37(0,04)$ & $-0,08(0,13)$ \\
\hline $\mathrm{PE}$ & $0,03(0,03)$ & $0,06(0,03)$ & $0,05(0,03)$ & $-0,06(0,03)$ & $-0,01(0,03)$ & $0,44(0,08)$ \\
\hline$\sigma_{a}^{2}$ & 0,27 & 0,30 & 0,28 & 0,0019 & 161,79 & 1,91 \\
\hline$\sigma_{f}^{2}$ & 0,90 & 0,80 & 0,89 & 0,0074 & 437,12 & 4,37 \\
\hline
\end{tabular}

${ }^{\mathrm{I}}$ Característica.

As correlações genéticas entre os escores visuais (Tab. 4) apresentam-se altas, sendo que a maior associação genética foi entre os escores $\mathrm{P}$ e $\mathrm{M}$ $(0,95)$; valores semelhantes foram reportados por Koury Filho et al. (2009). De forma geral, as correlações genéticas entre os escores visuais das diferentes metodologias desenvolvidas no Brasil apresentam-se altas (Cardoso et al., 2004; Weber et al., 2009; Koury Filho et al., 2010; Queiroz et al., 2011; Toral et al., 2011); esses resultados sugerem que existe uma dependência genética entre os escores. Assim, a seleção para qualquer dos escores trará mudanças genéticas no mesmo sentido para os outros, dificultando a seleção de forma independente.

Os três escores avaliados apresentaram a mesma tendência nas correlações genéticas com as características GPD, P18 e PE (Tab. 4), apresentando uma forte e moderada associação genética com o P18 e GPD, respectivamente; porém, a associação existente entre os escores e o PE foi próxima de zero com erros padrão altos. São poucos os trabalhos com estimação de correlações para essas características. Koury Filho et al. $(2009,2010)$ reportaram correlação semelhante entre E e Peso ao sobreano; correlações favoráveis e moderadas entre os escores e PE foram reportadas por Faria et al. (2009b); no entanto, as aqui apresentadas indicam que não existe associação genética entre elas.

A forte associação genética existente entre os escores e o peso do animal é esperada, uma vez que, na atribuição dos escores, faz-se uma estimação da área corporal do animal compreendida pelas dimensões de comprimento, largura e profundidade de costelas.

Os maiores erros padrão apresentados pelas correlações genéticas entre os escores e o PE podem ser consequência do menor número de informações para essa característica quando comparada com as outras (Tab. 2).

Levando em conta que as correlações genéticas entre os escores visuais e as características de GPD, P18 e PE apresentam a mesma tendência, e as altas correlações genéticas existentes entre os escores visuais (Tab. 4), pode-se pensar em juntar os escores mais correlacionados em um só. Essa foi a abordagem de outros países, os quais utilizam um ou dois escores para avaliar aspectos exteriores do animal, que podem ter relação com características de importância econômica (Phöcas e Laloe, 2004; Bouquet et al., 2010).

Os trabalhos encontrados na literatura que estimam correlações entre os escores E, P e M com características de crescimento são escassos (Faria et al., 2009a; Koury Filho et al., 2009). Isso sugere a realização de novos trabalhos, que permitam corroborar os resultados encontrados para a relação entre os escores visuais da metodologia EPM com outras características de importância econômica. 


\section{CONCLUSÕES}

A herdabilidade moderada dos escores EPM, as correlações genéticas com peso aos 18 meses e ganho de peso diário junto com a facilidade de mensuração sugerem que estes podem ser utilizados como critério de seleção para crescimento. As correlações genéticas entre os escores visuais indicam que os mesmos são controlados em grande parte pelo mesmo grupo de genes, sendo possível a inclusão de só um deles nos índices de seleção.

\section{AGRADECIMENTOS}

Ao Conselho Nacional de Desenvolvimento Científico e Tecnológico (CNPq) pelo apoio financeiro para o desenvolvimento desta pesquisa.

\section{REFERÊNCIAS}

AFOLOYAN, R.A.; PITCHFORD, W.S.; DELAND, M.P.; MCKIERNAN, W.A. Breed variation and genetic parameters for growth and body development in diverse beef cattle genotypes. Animal, v.1, p.13-20, 2007.

BOLIGON, A.A.; MERCADANTE, M.E.Z.; ALBUQUERQUE, L.G. Genetic associations of conformation, finishing precocity and muscling visual scores with mature weight in nelore cattle. Livest. Sci., v.135, p.238-243, 2011.

BOUQUET, A.; FOILLOUX, M.N.; RENAND, G.; PHÖCAS, F. Genetics parameters for growth, muscularity, feed efficiency and carcass traits of youngs beeg bulls. Livest. Sci., v.129, p.38-48, 2010.

CARDOSO, F.F.; CARDELLINO, R.A.; CAMPOS, L.T. Componentes de (co)variância e parâmetros genéticos de características pósdesmama em bovinos da raças angus. Rev. Bras. Zootec., v.33, p.313-319, 2004.

FARIA, C.U.; MAGNOBOSCO, C.U.; ALBUQUERQUE, L.G. et al. Utilização de escores visuais de características morfológicas de bovinos Nelore como ferramenta para o melhoramento genético animal. Planaltina, DF: Embrapa Cerrados, 2007. 40p.
FARIA, C.U.; MAGNOBOSCO, C.U.; ALBUQUERQUE, L.G. et al. Análise genética de escores de avaliação visual de bovinos com modelos bayesianos de limiar e linear. Pesqui. Agropecu. Bras., v.3, p.835-841, 2008.

FARIA, C.U.; KOURY FILHO, W.; MAGNOBOSCO, C.U. et al. Bayesian inference in genetic parameter estimation of visual scores in nellore beef-cattle. Genet. Mol. Biol., v.23, p.735-760, 2009a.

FARIA, C.U.; MAGNABOSCO, C.U.; ALBUQUERQUE, L.G. et al. Análise bayesiana na estimação de correlações genéticas entre escores visuais e características reprodutivas de bovinos Nelore utilizando modelos linear-limiar. Arq. Bras. Med. Vet. Zootec., v.61, p.949-958, 2009b.

FARIA, C.U.; PIRES, B.C.; VOZZI, A.P. et al. Genetic correlations between categorical morphological traits in nelore cattle by applying bayesian analysis under a threshold animal model. J. Anim. Breed. Genet., v.127, p.377-384, 2010.

HERRING, W.O; WILLIAMS, S.E.; BERTRAND, J.K. et al. Comparison of live and carcass equations predicting percentage of cutability, retail product weight, and trimmable fat in beef cattle. J. Anim. Sci., v.72, p.11071118, 1994.

JORGE JÚNIOR, J.; PITA, F.V.; FRIES, L.A. et al. Influência de alguns fatores de ambiente sobre os escores de conformação, precocidade e musculatura à desmama em um rebanho da raça Nelore. Rev. Bras. Zootec., v.30, p.1697-1703, 2001.

KOURY FILHO, W.; ALBUQUERQUE, L.G.; ALENCAR, M.M. et al. Estimativas de herdabilidade e correlações para escores visuais, peso e altura ao sobreano em rebanhos da raça Nelore. Rev. Bras. Zootec., v.38, p.2362-2367, 2009.

KOURY FILHO, W.; ALBUQUERQUE, L.G.; FORNI, S. et al. Estimativas de parâmetros genéticos para os escores visuais e suas associações com peso corporal em bovinos de corte. Rev. Bras. Zootec., v.39, p.1015-1022, 2010 . 
MAIWASHE, A.N.; BRADFIELD, M.J.; THERON, H.E.; VAN WYK, J.B. Genetic parameter estimates for body measurements and growth traits in south african bonsmara cattle. Livest. Prod. Sci., v.75, p.293-300, 2002.

MEYER, K. Wombat -a program for mixed models analyses in quantitative genetics by reml. J. Zhejiang. Univ. Sci., v.8, p.815-821, 2007.

PHÖCAS F.; LALOE, D. Genetic parameters for birthand weaning traits in french specialized beef cattle breeds. Livest. Prod. Sci., v.89, p.121-128, 2004.

QUEIROZ, S.A.; OLIVEIRA, J.A.; COSTA, G.Z.; FRIES, L.A. Estimates of genetic parameters for visual scores and daily weight gain in brangus animals. Animal, v.5, p.838-843, 2011.
$\mathrm{R}$ CORE TEAM. R: A Language and Environment for Statistical Computing. $\mathrm{R}$ Foundation for Statistical Computing, Vienna, Austria, 2010. URL http: //www.R-project.org/. ISBN 3-900051-07-0.

TORAL, F.L.; ROSO, V.M.; ARAÚJO, C.V.; REIS FILHO, J.C. Genetic parameters and response to selection for post-weaning weight gain, visual scores and carcass traits in hereford and hereford x nellore cattle. Livest. Sci., v.137, p.231-237, 2011.

WEBER, T.; RORATO, P.R.; LOPES, J.S. et al. Parâmetros genéticos e tendências genéticas e fenotípicas para características produtivas e de conformação na fase pré-desmama em uma população da raça aberdeen angus. Rev. Bras. Zootec., v.38, p.832-842, 2009.

YOKOO, M.J.; WERNECK, J.N.; PEREIRA, M.C. et al. Correlações genéticas entre escores visuais e caracterésticas de carcaçfa medidas por ultrassom em bovinos de corte. Pesqui. Agropecu. Bras., v.44, p.197-202, 2009. 